\title{
MECANISMO DE AÇÃO, EFEITOS FARMACOLÓGICOS E REAÇÕES ADVERSAS DA CEFTRIAXONA: UMA REVISÃO DE LITERATURA
}

\author{
MECHANISMS OF ACTION, PHARMACOLOGICAL EFFECTS, CLINICAL \\ STUDIES AND ADVERSE REACTIONS OF CEFTRIAXONE: A REVIEW OF \\ LITERATURE
}

MECANISMO DE ACCIÓN Y EFECTOS CLÍNICOS de ceftriaxona REACCIONES ADVERSAS: UNA REVISIÓN DE LA LITERATURA

Tiago Fernando Aragão Silva, Marcos Antônio de Araújo Filho, Maria dos Remédios Mendes de Brito e Rivelilson Mendes de Freitas

Recebido em 14/09/2009, Aceito em 25/09/2014.

RESUMO: A ceftriaxona é uma cefalosporina de terceira geração muito usada atualmente. É uma droga que age inibindo a síntese da parede microbiana, possuindo amplo espectro de ação contra bactérias gram-negativas. O objetivo do presente trabalho foi conduzir uma revisão de literatura sobre os aspectos farmacológicos da ceftriaxona, incluindo o mecanismo de ação que possa explicar os efeitos tóxicos e a toxicidade aguda e em longo prazo. Métodos: Para tanto foi realizada uma revisão da literatura usando as palavras-chaves ceftriaxona, reações adversas, farmacocinética e cefalosporinas, por intermédio do MEDLINE e LILACS. A busca inclui todos os artigos publicados no período entre 1985 a 2008. Resultados: Ainda existem muitas questões sem respostas sobre a farmacologia da ceftriaxona e a fisiologia dos efeitos tóxicos dessa substância. A simples descrição do mecanismo de ação é insuficiente para explicar todos os efeitos induzidos por este antibiótico. Conclusão: Existem poucas informações na literatura sobre a farmacologia e o mecanismo de ação da ceftriaxona que possam explicar seus efeitos adversos. São necessários mais estudos para que o profissional de saúde possa obter informações e conhecimento, a fim de combater os efeitos adveros 
SILVA, T.F.A., FILHO, M.A.A., BRITO, M.R.M.B., FREITAS, R.M.

REF-ISSN1808-0804 Vol.XI (3) 48-57, 2014.

decorrentes do tratamento com a ceftriaxona.

PALAVRAS ChAVES: Ceftriaxona, Reações Adversas, Farmacocinética, Cefalosporinas

ABSTRACT: Ceftriaxone is a third generation cephalosporin widely used today. It is a drug that inhibits the synthesis of the microbial wall, possessing broad spectrum of activity against gram-negative bacteria. The purpose of this study was to conduct a literature review on the pharmacological aspects of ceftriaxone, including the mechanism of action that may explain the toxic effects and acute and longterm. Methods: For this is a review of the literature using the keywords ceftriaxone, adverse reactions, pharmacokinetics and cephalosporins through the MEDLINE and LILACS. The search includes all articles published between 1985 to 2008. Results: There are still many unanswered questions about the pharmacology of ceftriaxone and physiology of its toxic effects. A simple description of the mechanism of action is insufficient to explain all the effects of this antibiotic. Conclusion: There is little information in the literature on the pharmacology and mechanism of action of ceftriaxone that may explain its adverse effects. Further studies are needed to ensure that health professionals may obtain information and knowledge in order to combat the adverse effects resulting from treatment with ceftriaxone.

KEY WORDS: Ceftriaxone, Adverse Reactions, Pharmacokinetics, Cephalosporins

RESUMEN: La ceftriaxona es una cefalosporina de tercera generación ampliamente utilizado hoy. Se trata de un fármaco que actúa inhibiendo la síntesis de la pared microbiana, que poseen amplio espectro de actividad contra las bacterias gramnegativas. El objetivo de este estudio fue realizar una revisión bibliográfica sobre los aspectos farmacológicos de ceftriaxona, incluyendo el mecanismo de acción que pueden explicar los efectos tóxicos y de toxicidad aguda ya largo plazo. Métodos: Para este fin, una revisión de la literatura se realizó utilizando la ceftriaxona palabras clave, reacciones adversas, farmacocinética y cefalosporinas a través del MEDLINE y LILACS. La búsqueda incluye todos los artículos publicados entre 1985 y 2008 Resultados: Todavía hay muchas preguntas sin respuesta sobre la farmacología de la ceftriaxona y la fisiología de sus efectos tóxicos. Una simple descripción del mecanismo de acción no es suficiente para explicar todos los efectos de este antibiótico. Conclusión: Hay poca información en la literatura sobre la farmacología y mecanismos de acción de ceftriaxona que podría explicar sus efectos 
SILVA, T.F.A., FILHO, M.A.A., BRITO, M.R.M.B., FREITAS, R.M.

REF-ISSN1808-0804 Vol.XI (3) 48-57, 2014.

adversos. Más estudios para el profesional de la salud pueden obtener información y el conocimiento con el fin de combatir los efectos de adveros se necesita tratamiento con ceftriaxona.

PALABRAS CLAVE: ceftriaxona, Reacciones adversas, Farmacocinética, cefalosporinas

\section{INTRODUÇÃO}

Os antibióticos são substâncias químicas produzidas

por microorganismos. Essas substâncias são capazes de destruir ou impedir o crescimento dos microorganismos patogênicos. Existem vários relatos médicos que descrevem o tratamento padrão, na antiguidade, para algumas infecções, como, por exemplo, o uso de terra e plantas, os quais provavelmente teriam a presença de microorganismos. Atualmente se tem conhecimento de várias drogas sintéticas que possuem efeito similar ao dos antibióticos. A cada dia que passa substâncias novas, destinadas ao uso como antimicrobianos são lançadas no mercado farmacêutico ${ }^{(1-}$ 2).

Após o lançamento da primeira cefalosporina para uso clínico, a cefalotina, de primeira geração, em 1964, inúmeros outros derivados cefalosporínicos surgiram e muitos outros ainda estão em fase de experimentação(3). As cefalosporinas são antibióticos enquadrados dentro do conceito de drogas betalactâmicas, pois possuem similaridade estrutural com as penicilinas. A cefalosporina C é extraída de um fungo do gênero Cephalosporium e, a partir desta, foram originados vários outros antibióticos que pertencem a esse grupo.

As cefalosporinas foram introduzidas na década de 60 , pelo fato de as penicilinas não serem mais efetivas contra estafilococos. São classificadas em cefalosporinas de $1^{a}$, $2^{a}, 3^{a}$ e $4^{a}$ geração. Atualmente o grupo mais abrangente das cefalosporinas é o das de terceira geração, devido às suas características farmacocinéticas e ao seu ampliado espectro de ação(3).

A ceftriaxona é uma cefalosporina de terceira geração muito usada atualmente. É uma droga que age inibindo a síntese da parede microbiana, possuindo amplo espectro de ação contra bactérias gramnegativas $^{(4)}$. Possui meia-vida elevada, alcançando elevados picos 
SILVA, T.F.A., FILHO, M.A.A., BRITO, M.R.M.B., FREITAS, R.M.

REF-ISSN1808-0804 Vol.XI (3) 48-57, 2014.

séricos. É indicada principalmente no tratamento de septicemia, infecções do trato respiratório e urinário, além do tratamento de meningite ${ }^{(3)}$. A ceftriaxona em geral é um antibiótico bem tolerado. Possuindo relativa estabilidade na presença de betalactamases, tendo uma alta afinidade pelas proteínas ${ }^{(5)}$.

\section{METODOLOGIA}

Neste contexto foi feita uma revisão bibliográfica sobre a ceftriaxona. Descreveram-se a estrutura química, mecanismo de ação, posologia, administração, indicação, farmacocinética, reações adversas, interações medicamentosas, mecanismo da toxicidade, contra-indicação e precauções. O mecanismo de ação foi relatado, a fim de justificar os efeitos farmacológicos e as possibilidades terapêuticas de reverter às principais complicações associadas ao uso da ceftriaxona. Para tanto, foi realizada uma revisão de 1985 até 2008 por meio do MEDLINE e LILACS, usando as seguintes palavras chaves: ceftriaxona, reações adversas, farmacocinética e cefalosporinas. Foram selecionados artigos publicados entre 1990 a 2008. Todos os artigos encontrados,

preferencialmente artigos de revisão, revisões sistemáticas e ensaios clínicos foram utilizados.

\section{ESTRUTURA QUÍMICA}

A estrutura química da ceftriaxona (figura 1) denominada segundo a IUPAC de (6R,7R)-7-(Z)-2(amino-4-tiazolil)-2[metoxiimino]acetamido-3-[(2,5diidro-6-hidroxi-2-metil-5-oxo-astriazin-3-il)tio]metil-8-oxo-5-tia-1azobiciclo[4,2,0]-oct-2-en-2-ácido carboxílico.

Figura 1: Estrutura química da ceftriaxona.<smiles>CON=C(C(=O)NC1C(=O)N2C(C(=O)O)=C(COc3nc(=O)c(=O)[nH]n3C)CS[C@H]12)c1csc(N)n1</smiles>

\section{MECANISMO DE AÇÃO}

As cefalosporinas interferem na síntese dos peptidoglicanos bacterianos após a ligação às proteínas de ligação de beta lactâmicos. Isso ocorre através da inibição da enzima de transpeptidação que forma ligações cruzadas das 
SILVA, T.F.A., FILHO, M.A.A., BRITO, M.R.M.B., FREITAS, R.M.

REF-ISSN1808-0804 Vol.XI (3) 48-57, 2014.

cadeias peptídicas ligadas ao arcabouço do peptidoglicano. O evento bactericida final consiste na inativação de um inibidor das enzimas autolíticas na parede celular levando à lise da bactéria( ${ }^{(4)}$.

\section{POSOLOGIA E ADMINISTRAÇÃO}

0 produto pode ser administrado via intravenosa (lentamente) ou intramuscular. A medicação deve ser mantida até dois dias após o desaparecimento dos sinais ou dos sintomas da infecção(12).

Em adultos e crianças acima de 12 anos: a dose usual é de 1 a $2 \mathrm{~g}$ de ceftriaxona em dose única diária. Em casos graves ou em infecções causadas por patógenos moderadamente sensíveis, a dose pode ser elevada para $4 \mathrm{~g}$, uma vez ao dia. Em recém-nascidos (abaixo de 14 dias) deve ser usada dose única diária de 20-50 mg/kg. Em lactentes e crianças (15 dias até 12 anos) utilizase dose única de $20-80 \mathrm{mg} / \mathrm{kg}$. Para crianças de $50 \mathrm{~kg}$ ou mais deve ser utilizada a posologia de adultos ${ }^{(6-7)}$.

No tratamento de meningite bacteriana de lactentes e crianças deve-se iniciar o tratamento com 100 $\mathrm{mg} / \mathrm{kg}$ em dose única diária. Para o tratamento da gonorréia causada por cepas produtoras e não produtoras de penicilase recomenda-se uma dose única de $250 \mathrm{mg}$. Na profilaxia no pré-operatório recomenda-se 1 a $2 \mathrm{~g}$ de ceftriaxona 30 a 90 minutos antes da cirurgia ${ }^{(6-8)}$.

\section{INDICAÇÃO}

A ceftriaxona é comercializada pelo laboratório Roche com o nome de Rocefin na Europa, Ásia e América Latina, pertence a uma classe química conhecida como cefalosporinas e é indicada para o tratamento da gonorréia endocervical não complicada, gonorréia uretral não complicada, infecção articular, infecção da pele e dos tecidos moles, infecção intra-abdominal, infecção óssea, infecção pélvica em mulheres, na profilaxia da infecção periorbitária, infecção urinária, meningite, pneumonia e septicemia ${ }^{(5-9)}$. 
SILVA, T.F.A., FILHO, M.A.A., BRITO, M.R.M.B., FREITAS, R.M.

REF-ISSN1808-0804 Vol.XI (3) 48-57, 2014.

\section{FARMACOCINÉTICA}

A seguir foram descritas as principais características

farmacocinéticas da ceftriaxona:

\section{Absorção}

A concentração plasmática máxima depois de dose única de $1 \mathrm{~g}$ IM é de cerca de $81 \mathrm{mg} / \mathrm{l}$ e é alcançada em 2 a 3 horas após a administração. A ceftriaxona caracteriza-se por apresentar uma meia-vida de eliminação extraordinariamente longa de aproximadamente 8 horas, em adultos sadios. As áreas sob as curvas de concentração plasmática versus tempo, após administração IM e IV são idênticas. Isto significa que a biodisponibilidade da ceftriaxona após administração IM é de $100 \%{ }^{(10)}$.

\section{Distribuição}

O volume de distribuição da cefriaxona é de 7 a 12 litros. A ceftriaxona mostrou excelente penetração tissular e nos líquidos orgânicos após dose de 1 a $2 \mathrm{~g}$. Alcança concentrações bem acima da concentração inibitória mínima contra a maioria dos patógenos responsáveis pela infecção, e são detectáveis por mais de 24 horas em mais de 60 tecidos ou líquidos orgânicos, incluindo pulmões, coração, fígado, vias biliares, amígdalas, ouvido médio, mucosa nasal, ossos, fluidos cérebro-espinhal, pleural, prostático e sinovial(4). A ceftriaxona liga-se de modo reversível à albumina, diminuindo a ligação com o aumento da concentração. Assim, para uma concentração plasmática < 100 $\mathrm{mcg} / \mathrm{ml}$, a ligação protéica é de $95 \%$, enquanto para uma concentração de $300 \mathrm{mcg} / \mathrm{ml}$, a ligação é de $85 \%$. Devido ao conteúdo mais baixo em albumina, a proporção de ceftriaxona livre no líquido intersticial é proporcionalmente mais alta do que no plasma.

\section{Biotransformação}

A ceftriaxona não é metabolizada sistemicamente, mas convertida a metabólitos microbiologicamente inativos pela microbiota intestinal ${ }^{(11-12)}$.

\section{Eliminação}

O clearance total do plasma é 10 a $20 \mathrm{ml} / \mathrm{min}$. O clearance renal é 5 a $12 \mathrm{ml} / \mathrm{min}$. Em adultos cerca de 50 a $60 \%$ de ceftriaxona é excretada sob 
SILVA, T.F.A., FILHO, M.A.A., BRITO, M.R.M.B., FREITAS, R.M.

REF-ISSN1808-0804 Vol.XI (3) 48-57, 2014.

a forma inalterada na urina, enquanto 40 a $50 \%$ são excretados sob a forma inalterada na bile. A meia-vida de eliminação em adultos sadios é de aproximadamente 8 horas $^{(4)}$.

\section{REAÇÕES ADVERSAS}

Os principais efeitos adversos são os distúrbios gastrointestinais, as alterações hematológicas e as reações cutâneas. Os distúrbios gastrointestinais incluem diarréia, náuseas, vômitos, estomatite e glossite. As alterações hematológicas incluem eosinofilia, leucopenia, granulocitopenia, anemia hemolítica e trombocitopenia. As reações cutâneas são: exantema, dermatite alérgica, prurido, urticária, edema e eritema multiforme. Existem ainda os efeitos colaterais raros, que são cefaléia, tontura, elevação das enzimas hepáticas, sedimento sintomático de ceftriaxona cálcica na vesícula biliar, oligúria, aumento da creatinina sérica, micose do trato genital, tremores e reações anafiláticas ${ }^{(5,13-14)}$.

\section{INTERAÇÕES MEDICAMENTOSAS}

Até o momento, não se observou quaisquer alterações da função renal após administração simultânea de doses elevadas de ceftriaxona e potentes diuréticos, como a furosemida, em altas doses. Não existe também qualquer evidência de que a ceftriaxona aumenta a toxicidade dos aminoglicosídios ${ }^{(15)}$.

A cefttriaxona não apresentou efeito similar ao provocado pelo dissulfiram após administração de álcool. A ceftriaxona não contém o radical $\mathrm{N}$-metiltiotetrazol que está associado a uma possível intolerância ao álcool e a sangramentos observados com outras cefalosporinas. O probecinida não têm influência sobre a eliminação da ceftriaxona. Em estudos in vitro efeitos antagônicos foram observados com o uso combinado de clorafenicol e ceftriaxona. A ceftriaxona não deve ser adicionada a soluções que contenham cálcio como a solução de hartmam ou solução de Ringer. É incompatível com amsacrina, vancomicina e fluconazol $^{(15)}$.

\section{MECANISMO DA TOXICIDADE}

As cefalosporinas têm a capacidade de sensibilizar o indivíduo e podem desencadear uma variedade de reações de hipersensibilidade que são idênticas às das penicilinas 
SILVA, T.F.A., FILHO, M.A.A., BRITO, M.R.M.B., FREITAS, R.M.

REF-ISSN1808-0804 Vol.XI (3) 48-57, 2014.

incluindo anafilaxia, febre, erupções cutâneas, nefrite e anemia hemolítica. A irritação local pode produzir dor intensa após injeção intramuscular e tromboflebite após injeção intravenosa. Foi demonstrada a ocorrência de nefrotoxicidade ${ }^{(6)}$.

A resistência às cefalosporinas aumentou em decorrência das betalactamases codificadas por plasmídios ou cromossomos. Também, pode ocorrer resistência se houver menor penetração do fármaco em conseqüência das alterações nas proteínas da membrana externa ou de mutações nas proteínas dos sítios de ligação ${ }^{(6)}$.

\section{CONTRA-INDICAÇÕES E PRECAUÇÕES}

Não deve ser usada por pacientes com reconhecida hipersensibilidade aos antibióticos do grupo das cefalosporinas. Em pacientes hipersensíveis à penicilina deve-se levar em conta a possibilidade de reações alérgicas cruzadas $^{(16)}$.

Embora as pesquisas préclínicas não tenham revelado efeitos mutagênicos e teratogênicos, a ceftriaxona não deve ser usada durante a gravidez (principalmente nos 3 primeiros meses), a não ser que seja absolutamente necessário. Como a ceftriaxona é excretada no leite em baixas concentrações, é recomendável cuidado em mulheres que amamentam $^{(4)}$.

Como ocorre com outras cefalosporinas, a ocorrência de choque anafilático não pode ser afastada mesmo na ausência antecedentes alérgicos. A ocorrência de choque anafilático exige imediata intervenção ${ }^{(6)}$.

Em casos raros o exame ultrassonográfico da vesícula biliar revelou imagens de sedimento que desaparecem com a descontinuação ou conclusão da terapêutica com ceftriaxona, então, recomenda-se tratamento clinico conservador, mesmo nos casos em que tais achados se acompanham de sintomatologia dolorosa ${ }^{(10)}$.

Estudos in vitro demonstraram que a ceftriaxona, como outras cefalosporinas, pode deslocar a bilirrubina da albumina sérica. Sendo assim, a ceftriaxona não é recomendada para neonatos, especialmente prematuros que apresentem risco de desenvolver encefalopatia devido à hiperbilirrubinemia(17). 
SILVA, T.F.A., FILHO, M.A.A., BRITO, M.R.M.B., FREITAS, R.M.

REF-ISSN1808-0804 Vol.XI (3) 48-57, 2014.

Durante tratamentos

prolongados, deve-se fazer controle regular do hemograma. No caso de insuficiência hepática e renal graves e concomitantes, deve-se determinar a concentração plasmática de ceftriaxona a intervalos regulares. Dados não indicam efeitos adversos em pessoas que trabalham com máquinas ou veiculos automotores ${ }^{(16)}$.

É recomendável não injetar mais que $1 \mathrm{~g}$ em cada glúteo. Além disso, devem-se evitar doses acima de 4 g/dia nas administrações intravenosas. E a apresentação com lidocaína nunca deve ser administrada por via intravenosa ${ }^{(1-2)}$.

\section{CONCLUSÃO}

Portanto, a ceftriaxona é um antibiótico muito utilizado no tratamento de infecções causadas por bacilos gram-negativos. No entanto, 0 uso clínico de antibióticos exige o conhecimento de uma série de noções básicas e princípios gerais que permitam o seu emprego racional e a obtenção dos resultados satisfatórios. O uso incorreto de antibióticos pode induzir à resistência bacteriana, dentre outros problemas. No caso da ceftriaxona, uma resistência bacteriana poderia causar um aumento na proliferação dos microorganismos sensíveis a esse antibiótico. Então, é essencial que o profissional de saúde conheça os efeitos farmacológicos e toxicológicos provocados pelo uso da ceftriaxona. Esse artigo traz importantes considerações sobre o mecanismo de ação e sobre as propriedades farmacocinéticas e toxicológicas da ceftriaxona necessárias para seu uso seguro e eficaz no tratamento de infecções bacterianas.

\section{REFERÊNCIAS BIBLIOGRÁFICAS}

1. BERGOGONE-BÉRÉZIN, E. Activité antibactérienne de la ceftriaxone. La Revue de Médecine Interne. 1985;6:178-86.

2. KLERK, G.J., et al., A randomised, multicentre study of ceftriaxone versus standard therapy in the treatment of lower respiratory tract infections. International Journal of Antimicrobial Agents. 1999;12:121-27.

3. LOPES, H.V. Cefalosporinas de terceira geração: propriedades limitações e indicações terapêuticas. Arq. Med. ABC. 1990;13:30-32.

4. RANG, H.P., DALE, M.N. Farmacologia. 6th ed., Rio de Janeiro, Elsevier; 2008.

5. SILVA, P. Farmacologia. 7th ed., Rio de Janeiro: Guanabara Koogan; 2006. 
SILVA, T.F.A., FILHO, M.A.A., BRITO, M.R.M.B., FREITAS, R.M.

REF-ISSN1808-0804 Vol.XI (3) 48-57, 2014.

6. KATZUNG, B.G. Farmacologia Básica e Clínica. 9th ed., Rio de Janeiro: Guanabara Koogan; 2006.

7. BÉGUÉ, P., et al. Evaluation de la ceftriaxone et du céfotaxime dans l'infection bactérienne sévère en pédiatrie: étude multicentrique. Médecine et Maladies Infectieuses. 1998;28:300-06.

8. KOMOROSKI, E.M., et al., Single-dose intramuscular ceftriaxone for the treatment of uncomplicated cystitis in children and adolescents. Current Therapeutic Research. 1999;60:580-94.

9. ROSENBERG, J.M., et al., Single-dose ceftriaxone treatment of urinary tract infections. Annals of Emergency Medicine. 1985;14:970-72.

10. COSTA, D., et al. Colelitíase associada ao uso de ceftriaxona. Revista da Sociedade Brasileira de Medicina Tropical. 2005;38:521-523.

11. MACHADO, A.R Pseudolitíase biliar após terapia por ceftriaxona: relato de caso. Pediatria 2001;23:346-8.

12. TSATSAKIS, A.M., et al., Comparative pharmacokinetics of ceftriaxone after periincisional and intravenous administration in patients undergoing abdominal surgery. European Journal of Pharmaceutical Sciences $1997 ; 5: 243-51$.

13. CERAN, C., et al., Ceftriaxone-associated biliary pseudolithiasis in paediatric surgical patients. International Journal of Antimicrobial Agents. 2005;25:256-59.

14. CHIAPPINI, E. Results of a 5-year prospective surveillance study of antibiotic resistance among Salmonella enterica isolates and ceftriaxone therapy among children hospitalized for acute diarrhea. Clinical Therapeutics. 2002; $24: 1585-94$.

15. FERREIRA, L.L.M., et al. Colonização e resistência antimicrobiana de Streptococus pneumoniae isolado em nasofaringe de crianças com rinofaringe aguda. J. Pediatria. 2001;77:227-34.

16. FERRAZ, A.A.B., et al. Profilaxia antimicrobiana na cirurgia bariátrica. Rev. Col. Bras. Cir. 2007;34:73-77.

17. BERGOGONE-BÉRÉZIN, E. Ceftriaxona: farmacocinética e farmacodinâmica; aplicações no tratamento da otite média aguda criança. Pediatria Mod. $1999 ; 35: 422-21$. 